\title{
A Sociotechnical Mechanism for Online Support Provision
}

\author{
Joshua Introne \\ Michigan State University \\ East Lansing, MI \\ jintrone@msu.edu
}

\author{
Bryan Semaan \\ Syracuse University \\ Syracuse, NY \\ bsemaan@syr.edu
}

\author{
Sean Goggins \\ University of Missouri \\ Columbia, MO \\ GogginsS@missouri.edu
}

\begin{abstract}
Social support can significantly improve health outcomes for individuals living with disease, and online forums have emerged as an important vehicle for social support. Whereas research has focused on the delivery and use of social support, little is known about how these communities are sustained. We describe one sociotechnical mechanism that enables sustainable communities to provide social support to a large number of people. We focus upon thirteen disease-specific discussion forums hosted by the WebMD online health community. In these forums, small, densely connected cores of members who maintain strong relationships generate the majority of support for others. Through content analysis we find they provide informational support to a large number of more itinerant members, but provide one another with community support. Based on these observations, we describe a sociotechnical mechanism of online support that is distinct from nonsupport oriented communities, and has implications for the design of self-sustaining online support systems.
\end{abstract}

\section{Author Keywords}

Online Social Support; Social Network Analysis; Online Communities

\section{ACM Classification Keywords}

K.4.3 [Computers and Society]: Organizational Impacts Computer-Supported cooperative work.

\section{INTRODUCTION}

Social support can play an important role in health outcomes for individuals with chronic diseases, impacting an individual's mental and physiological well-being, as well as improving clinical outcomes for a variety of diseases [49]. Despite this, there is no defined role for social support in the public healthcare system [57].

Perhaps in response to this gap, a significant amount of social support activity is now transacted online, leading researchers and service providers alike to investigate the

Permission to make digital or hard copies of all or part of this work for personal or classroom use is granted without fee provided that copies are not made or distributed for profit or commercial advantage and that copies bear this notice and the full citation on the first page. Copyrights for components of this work owned by others than the author(s) must be honored. Abstracting with credit is permitted. To copy otherwise, or republish, to post on servers or to redistribute to lists, requires prior specific permission and/or a fee. Request permissions from Permissions@acm.org. CHI'16, May 07 - 12, 2016, San Jose, CA, USA

Copyright is held by the owner/author(s). Publication rights licensed to ACM. ACM 978-1-4503-3362-7/16/05 ..\$15.00 .

DOI: http://dx.doi.org/10.1145/2858036.2858582 design and function of these systems. Whereas social support transacted online is important for people living with disease [7,46,47], little is known about how these communities are sustained. Here, we describe a sociotechnical mechanism that enables some support forums to address the health support needs of many people, and also to be self-sustaining. Our aim is to inform sociotechnical design efforts to create more resilient, effective platforms for online health support provision.

We focus upon publicly available posting data scraped from a set of disease specific forums hosted by the WebMD online health service. We find that many of these forums exhibit a core-periphery [5] structure, in which a small, highly inter-connected group of active, long-term users provide much of the informational support requested by a much larger itinerant population of peripheral users. Coreperiphery structures have been observed in other social platforms (e.g. $[14,25,56]$ ); what is unique about their existence here is their role in creating a mechanism for large-scale, sustainable social support. Specifically, we find that cores consist of very small groups of online friends who invest enormous amounts of time in providing one another with community support (defined below) [2]. In so doing, they build the capacity to provide informational support (also defined below) [2] to a broader group of support seekers. The system is sustained, in part, because core members are drawn back to the site by the relationships they develop with one another.

We present our analysis framed by a series of research questions, as follows:

1) What is the social organization of an online health support community?

To pursue this question, we must infer relationships between users from the conversational interactions they have online. This is not straightforward because our data omits conversational replies. Thus, we introduce a simple rule-based algorithm to infer the intended target of each message based on conventions posters use when addressing each other. We then segregate the population into core and peripheral users, noting that that there is a very large class of "extra-peripheral" users - that is, people who post very infrequently.

2) How is the task of providing social support allocated amongst community members in these systems?

After classifying users, we examine the message traffic within and between classes. We find that the core is 
responsible for the majority of responses to the other classes. We analyze messages from the core to determine the amount and kinds of support they contain, and find significant differences in the levels of community and informational support provided to the three classes of users.

3) By what mechanism might an online community sustain itself while providing social support to a large population?

The preceding analysis suggests a mechanism that enables some online support forums to sustain themselves while providing support to a large population of users. We propose that a small group of individuals form strong online relationships, providing one another with community support. As a result of the amount of time these individuals invest in the forum, they are available to respond to many peripheral users. This responsiveness in turn encourages newcomers to post questions to the forum.

In the following, we present related research on online support and sustainable social platforms, and then elaborate upon the preceding analysis. Finally, we conclude with a brief discussion about the implications of our work and where it may lead in the future.

\section{BACKGROUND}

Social support is a multi-dimensional construct covering the various kinds of support individuals provide one another. Various taxonomies of support have been proposed, but three dimensions appear frequently in literature on online support: community / companionship support (membership in a group with shared interests), emotional support (expressions of caring and comfort), and informational support (provision of knowledge or advice) [2,17,52]. These dimensions have been found to be correlated, yet distinct, and their expression depends both upon context and the nature of the relationships involved [17,55].

Giving and receiving social support has been demonstrated to have a range of profound impacts on the physical and mental well-being of individuals. Social support has been associated with improved clinical outcomes for a variety of conditions (e.g. [7,46,47]). Various reasons have been offered to explain this effect. Researchers have shown, for instance, that social support reduces the production of stress hormones, leading to improved immune response and reduction in stress-related disease [49]. Social support can assist with various forms of self-management, such as adherence to diet and medication regimens [22]. Informational support clearly has instrumental value in helping individuals manage their conditions [12], but its exchange can also be part of a coping process [50].

While the mechanisms and function of social support are current and active areas of inquiry, its value has long been recognized, leading to explicit calls for policies and clinical guidelines that mobilize social support [26,32]. Online health forums may viewed as an organic response to this need [49].

An online health forum is a place where people with medical conditions can go to find social support that complements the services provided by professional health care providers $[11,23,28,35]$. However, this description obscures the vast diversity found within online support communities. For example, Wang et al. [52] found that members in a breast cancer support community clustered into seven different groups according to the kinds of support sought and provided and, consistent with Wang et al. [54], found that these groupings were also predictive of member tenure and engagement. Bambina [2] found that members in a breast cancer support forum segregate into "takers" who receive support and "providers" who deliver it, and that the types of support sought and provided vary between these groups.

However, few analyses specific to online social support for medical issues have sought to articulate the ways in which different roles divide the "work" of providing large-scale social support in a coherent manner. Such mechanisms have been described in other online communities. For instance, work on Usenet-based discussion groups has revealed that individuals segregate into "questioner" and "answerer" roles, suggesting that the two populations sustain one another in symbiotic fashion [24,56]. A commonly observed pattern is for the work of a given site to be divided between a small committed core of users who have greater responsibility or authority, and a much larger, less critical periphery [13,41]. For example, Crowston et al. [14] described how open-source software communities are organized according to a core-periphery structure, with different roles and responsibilities for coordinating and developing software. Similar obsrevations have been made about Wikipedia and other online communities [6,10,37]. Bamabina also found evidence for a core-periphery social network organization in a breast cancer forum [5].

How such intra-community stratification might give rise to sustainable communities is a nuanced topic. Butler and colleagues describe community sustainability (and the related construct of resilience) as the ability of an online social system to buffer minor variation in site activity and content while maintaining membership levels [8,9]. The term "critical mass" has been used loosely to describe the point at which a site has developed sufficient resources to weather such minor variation $[29,45]$. Yet the decay of Wikipedia [48] over time suggests there is more at work than the simple accretion of resources; different sociotechnical constraints may come into play over time, and have varying impacts on different types of contributors. For example, Ren et al. [40] speculate that bond-based communities might grow with an initial network of friendships, but ultimately stagnate because, once such cliques form, they are not welcoming to newcomers.

Gleave et al. [24] introduced the term "role ecology"evocative of predator-prey dynamics - to describe the dynamic interplay between populations of users who play different roles within a given platform. From this perspective, a sustainable system is one in which interactions between and among populations with different 
roles stabilize while also enabling the system under consideration to continue doing its work-be that creating a living encyclopedia of all human knowledge, building open-source software, or providing social support to individuals with health problems.

We apply this framing here. We seek to describe how different roles may interact to create a sustainable online platform for support delivery. We do not claim that the mechanism we identify is the only possible sociotechnical organization for a sustainable online health support system, but it may be unique to these systems for two reasons. First, a stream of individuals with important and emergent information needs continually drives traffic to health support forums. Second, long-term sufferers of chronic disease can feel alienated within their local communities, and so are likely to seek the company of other sufferers online $[18,20]$. These two factors differentiate online health support from more generic question and answer forums, and may explain the dynamics we describe.

\section{WEBMD}

WebMD is a popular online health resource (as of January 2016, Alexa ${ }^{1}$ ranked WebMD.com as the $105^{\text {th }}$ most popular site in the US, and second most popular health site, behind NIH.gov, globally [58]). In addition to providing news, resources to assist with self-diagnosis and a nationwide directory of medical professionals, WebMD also hosts an array of topic-specific question \& answer forums. Top-level posts in these forums are questions and replies are answers, but a large amount of conversational interaction occurs within this context. Fifty-five of these forums are currently "featured," which means that WebMD experts participate in discussions, and that spam is deleted.

Our investigation focuses upon a set of forums in the WebMD online health community, covering roughly five years of data $(\sim 1$ million posts, $\sim 227 \mathrm{k}$ posters $)$ scraped from web-forums hosted on the site. We begin our analysis with the 55 "featured" forums on the site, and then narrow our focus to thirteen forums that name a specific disease or mental disorder and exhibit the structural property of a tightly connected core group of users. We narrow our analysis in this manner for two reasons. First, prior research has documented the important role social support plays in improving health outcomes for populations with specific chronic health conditions, and we are primarily interested in these populations. Many forums on WebMD cover nonmedical issues (e.g "Raising Fit Kids", "Food and Cooking", "Parenting") or very broad health concerns (e.g. "Gynecology", "Cholesterol management", "Men's health"), and thus are of questionable relevance. Second, we observe that core-periphery structures are widespread both in the forums we examine and other social-media. While it is possible and important to examine the

\footnotetext{
${ }^{1}$ Alexa is a commercial tool used to rank various Internet sites. It can be considered a rough indicator of site popularity in the absence of a standardized index.
}

exceptions to this pattern, we seek to understand its predominance first.

\section{THE SOCIAL STRUCTURE OF WEBMD}

Different kinds of conversations may be found in online support communities. As an illustrating case contrast two posts taken from the diabetes forum hosted at WebMD:

Post 1: Good Morning! Dorian, hope you'll feel better! Yeah...the loss of weight is also nice, but not worth getting sick. Oatmeal helped me when I had an upset stomach, AFTER I started feeling better.

Alpharuby, have a great lunch and day with your wife. Today's exercise is total house cleaning!! Have a good day everyone! I found out it will be in the 60's today, so I'm going for a long walk, too.

Post 2: Steroids raise blood sugar. I have experienced herniated/deteriorating disk pain, was given steroids and my blood sugar went up into the 300s. NSAIDS have worked for me and they have not raised my blood sugars. However continued unchecked pain will raise both blood sugar and blood pressure. Work with your MD and diabetic dietician.

Post 1 is from a conversation between three long-time users. Even out of context, the post suggests that it is part of a longer, extended interaction; the poster is keeping track of the other members, mixing a small bit of information about diabetic maintenance routines ("Oatmeal helped me when I had an upset stomach") with a larger portion of more trivial information informing others about the poster's personal activities ("Today's exercise is total house cleaning"). The latter post, on the other hand, is devoid of personal trivia, and reveals no evidence of an existing relationship.

These two posts illustrate an important difference in the kinds of conversations found in the WebMD forums. Consistent with the analysis by Bambina [2], many forums appeared to have a small group of highly active members with strong relationships. These individuals mention each other when talking to third parties, enquire after one another's well-being and daily routines, and comment on one another's absences when they occur. Yet they are not an exclusive group - they talk to other members who are not part of the group, offering advice and support to firsttime and one-time posters. More formally, individual forums conform to a core-periphery structure [5], consisting of a densely connected core of users, surrounded by a periphery of individuals who are only weakly connected to each other, but do interact with the core. We sought to provide quantitative support for this observation by first extracting user relationships and then attempting to fit the resultant network to a standard core / periphery model.

\section{Inferring replies}

The basis for our inferences about relationships is the existence of replies from one user to another. Any given post may be a reply to one or more individuals appearing previously in the thread, or it may not be a reply to anyone. 
Unfortunately, prior to a redesign that took place in February 2010, the UI offered no affordances for directing a reply, and this functionality was rarely used after it was introduced. This presents a challenging problem, closely related to research focused on automatically extracting reply sequences from unstructured forums $[1,33,51,53]$.

There is no single, agreed upon metric for reporting the performance of algorithms that extract reply structures. As with other information retrieval studies, it is common to report precision (in a given set of items, the proportion of correctly labeled positives to all labeled positives) and recall (in a given set of items, the proportion correctly labeled positives to all actual positives). These values are sometimes summarized as the $F_{1}$ score, which is the harmonic mean of precision and recall $(2 *(P * R /(P+$ $R)$ ) [38]. It is also common to report accuracy, which is the proportion of correctly labeled items to all items.

Where reported, $F_{1}$ scores $^{2}$ for recent general approaches range between $.40-.58[1,53]$ against different datasets, and accuracy scores between .47 - .78 [1,34,51]. We note that the domain used in [34] was an online health forum, and the authors reported maximum accuracy scores of .64.

However, our task is somewhat different than prior work on extracting reply structures. First, we do not care about precisely which post any given post is a response to, but rather the poster to which a given response is targeted. Second, individual posts may in fact respond to multiple users (e.g. "thanks everybody!"). Given the generally poor performance of existing machine learning procedures at extracting reply-to sequences, as well as the unique nature of the task at hand, we developed our own heuristic procedure.

Similar to the observations made in [34], we observed that posters frequently address one another by some kind of signifier. The referents used by posters in responding to one another did not always correspond to forum handles, and even varied over time (making it difficult to apply named entity recognition procedures, which depend upon a list of known entities). Nonetheless, naming conventions followed a variety of regular patterns. We found the following useful for establishing the relationship between handles and referring names.

- Conventional shortenings-Respondents often shorten handles in predictable ways, such as dropping numeric suffixes (e.g. Mia14875 may appear as simply Mia) or splitting handles at internal capitals (AprilJune may be referred to as April or June)

- Signatures-Posters often closed their posts with an easy to distinguish signature. Signatures were generally the last word in the post, followed some kind of punctuation, and were often capitalized.

\footnotetext{
${ }^{2}$ On reply-to relationships, not accounting for the similarity of thread hierarchies. This is the "edge" metric described by $[51]$.
}

- Self-identifiers-Posters occasionally introduced themselves formally, e.g. "Hi, my name is John"

- Frequent references - In cases where it was hard to determine the target of a reference, inferences could be made by looking at the statistical co-occurrence between references of the form "Hi, John," and poster handles over multiple threads.

Based on these observations, we developed a rule-based pattern matching procedure. First, using the preceding observations, we collected all possible references that may be used to refer to individuals in the forum. This yields a dictionary in which each handle is associated with one or more possible referents. The algorithm for inferring references then proceeds by comparing the words in the post to the list of known referents and previously seen authors in a thread. The details of this algorithm (and executable code) are available in a public code repository hosted at https://github.com/jintrone/WebMDChi16.

To evaluate the algorithm's performance, two coders manually coded 869 posts randomly drawn from the top ten most active forums (one hundred posts were randomly drawn from the replies in each of the 10 forums, but 131 posts were dropped from this set due to a clerical error). The coders were asked to identify explicit replies to or mentions of any posters appearing previously in a thread. The coders split the data, and then reviewed one another's codes, working together to resolve any disagreements. Note that because no training set is necessary for our algorithm, the entire set was available for testing.

We evaluate precision and recall against the set of all possible reply edges for each post. For instance if post 4 in a given thread is authored by Amy and appears after prior posts by Amy, Brad, and Carla, than there are two possible replies (to Brad and/or Carla; it is not meaningful in our context for a person to reply to themselves). If our algorithm predicts a reply to Brad, but the reply is actually to both Brad and Carla, than that would count as one true positive and one false negative.

The algorithm achieves precision and recall values of .80 and .81 respectively, for an overall $\mathrm{F}_{1}$-measure of .8 , and an accuracy of .90 . Thus the procedure performs substantially better than existing machine learning approaches do at extracting reply sequences. Furthermore, and as we will discuss, relationship strength (as we have defined it) tends to be distributed according to a power law, so there are many more opportunities for the algorithm to identify strong pairs than weak pairs. Thus, we expect that the errors in introduced by the algorithm will tend to preserve strong relationships, but may penalize weaker relationships.

\section{Inferring relationships}

While directed replies offer a basis for inferring social ties between community members, these inferences are highly subjective [30]. However, strong relationships tend to develop over a long series of dyadic interactions between the poster and addressee [27]. As an example, in the posts 
presented at the beginning of the section, the first is an instance of a post that appears in a long sequence of dyadic interactions, whereas the second post is a response to a first time poster.

Thus, following established practice in social network research [27], we use dyadic interactions, rather than individual replies, as our unit of evidence of a relationship. For our purposes, we consider a dyadic interaction to be the smallest perceivable unit of reciprocal communication [43] in which an interlocutor A posts a reply to $\mathrm{B}$, and then $\mathrm{B}$ posts a reply to $\mathrm{A}$ in the same thread. Restricting evidence for dyadic interactions to individual threads (rather than splitting them over multiple threads) guarantees that individuals are engaged in reciprocal communication, rather than simply talking at one another. Note that top-level posts are sometimes directed to specific individuals, but this is both infrequent and difficult to detect, and so we restrict our analysis to replies only.

Using this definition of a dyadic interaction, we constructed undirected, weighted social-networks for each individual forum over the duration of the collection period, where link weights corresponded to the number of dyadic interactions between two individuals. Across the forums, an average of $18 \%$ ( $41 \mathrm{k}$ posters over all 55 forums; median=17\%) of all posters had one or more dyadic interactions. These values are distributed normally $(W=.95, p<.05)$, and the proportion of dyadic interactions was independent of the total number of posters in a forum.

Within most of the constructed networks, edge weights fit a long tailed distribution. This is consistent with the majority of findings in online social network analysis (e.g. [19]), and indicates that a handful of people in the WebMD forums develop very strong relationships, while the vast majority of people have substantially weaker relationships.

As an approach to distinguishing between strong and weak relationships, we can estimate the probability that any dyad in a given forum will have continued interactions given that they have already had $X$ interactions as follows. Let $G(N, E)$ be the weighted, undirected graph representing all relationships $E$ and users $N$ in a given forum (where a relationship exists if there has been one or more dyadic interactions between connected users). Each edge $e \in E$ is assigned a weight $w$, which is the number of dyadic interactions the connected users have had. Let $E^{w>X}$ be the set of edges with weight greater than $\mathrm{X}$. Thus, we estimate the probability that any pair of users will have additional interactions once they have had $\mathrm{X}$ interactions as:

$$
P(w>X)=\frac{\left|E^{w>X}\right|}{\left|E^{w \geq X}\right|}
$$

This allows us to make informed judgments about when a series of dyadic interactions are likely to blossom into something greater. This is important when attempting to establish the core periphery structure of the overall social network, as described below.

\section{Core and Periphery}

Networks in many different domains exhibit what is often referred to as a core-periphery structure, describing a situation in which there is a densely inter-connected core, surrounded by a sparsely connected periphery $[5,15,16]$. More intuitively, members of the core tend to know and have relationships with all other members in the core, where as members of the periphery know members of the core, but are unlikely to know one another. In social networks, the detection of core-periphery structures is distinct from community detection because distinct communities tend to be connected only weakly or not at all, whereas individuals in a densely connected core may also be connected to other individuals in the weakly connected periphery.

Core-periphery structures are common in natural systems [15], but have also been found in many online communities, including open-source software [14,36], Wikipedia [25], discussion groups [21], and online support [2]. Core members often have higher status within a given community, more responsibility, and generate most of the activity in the system.

Borgatti and Everett [5] offered a procedure for detecting the existence of core-periphery structures by first defining an ideal prototypical core-periphery network, and then seeking a classification of nodes in an actual network to best match the ideal. In the ideal networks they proposed, the nodes in the core are fully connected, nodes in the periphery are not connected, and connections between core and periphery vary depending upon one's assumptions. Following Crowston et al. [14], we assume nothing about the presence or absence of links between core and periphery (a mathematical description of this idea may be found in [5:383]).

Borgatti and Everett [5] recommended applying Pearson's correlation in weighted networks to evaluate the fit of a given core-periphery classification of nodes. However, because edge weights in many of our networks fit a longtailed distribution, this fitness function excludes moderately weighted edges from the core even though they are stronger than the vast majority of edges in the graph. Thus, we applied equation (1) above to determine a cutoff weight for each forum by choosing the minimum weight at which the probability of future interactions was greater than 0.5 .

To calculate core-periphery structures, we followed the procedure described in [16], using a low-rank approximation to the target matrix first to sort nodes by their "coreness," followed by local hill climbing (code is available at https://github.com/jintrone/WebMDChi16). Unlike the implementation in UCINet [4], this approach offers faster performance with deterministic outcomes.

There is no accepted statistical test of significance for the procedure described by Borgatti and Everett [5], and so, following their recommendations, we use a correlation of $\mathrm{R}=.60$ as a cutoff for determining whether or not a network is sufficiently core-like to be considered an acceptable fit. 


\begin{tabular}{lcccc} 
& \multicolumn{4}{c}{ Top Level Posts } \\
\cline { 2 - 5 } Name & Core & Periph. & X-Periph. & Staff \\
\cline { 2 - 5 } Dibromyalgia & $7 \%$ & $6 \%$ & $2 \%$ & $0 \%$ \\
Mubetes & $6 \%$ & $5 \%$ & $5 \%$ & $1 \%$ \\
Breast Cancer & $5 \%$ & $12 \%$ & $5 \%$ & $1 \%$ \\
Lupus & $4 \%$ & $7 \%$ & $6 \%$ & $1 \%$ \\
Epilepsy & $6 \%$ & $11 \%$ & $9 \%$ & $1 \%$ \\
HIV and AIDS & $4 \%$ & $9 \%$ & $8 \%$ & $1 \%$ \\
Asthma & $0 \%$ & $12 \%$ & $15 \%$ & $1 \%$ \\
ADD and ADHD & $3 \%$ & $8 \%$ & $17 \%$ & $2 \%$ \\
Alzheimer's & $0 \%$ & $7 \%$ & $23 \%$ & $2 \%$ \\
Hepatitis & $2 \%$ & $3 \%$ & $5 \%$ & $2 \%$ \\
Parkinson's & $2 \%$ & $7 \%$ & $16 \%$ & $2 \%$ \\
Osteoporosis & $3 \%$ & $7 \%$ & $20 \%$ & $1 \%$ \\
\hline AVERAGE & $0 \%$ & $8 \%$ & $17 \%$ & $4 \%$ \\
\cline { 2 - 5 } & $3 \%$ & $8 \%$ & $11 \%$ & $1 \%$
\end{tabular}

\begin{tabular}{cccc}
\multicolumn{4}{c}{ Replies } \\
\hline Core & Periph. & X-Periph. & Staff \\
\hline $42 \%$ & $33 \%$ & $8 \%$ & $2 \%$ \\
$50 \%$ & $21 \%$ & $6 \%$ & $5 \%$ \\
$37 \%$ & $33 \%$ & $7 \%$ & $1 \%$ \\
$40 \%$ & $25 \%$ & $14 \%$ & $3 \%$ \\
\hline $22 \%$ & $37 \%$ & $10 \%$ & $4 \%$ \\
\hline $43 \%$ & $25 \%$ & $7 \%$ & $2 \%$ \\
\hline $34 \%$ & $25 \%$ & $9 \%$ & $4 \%$ \\
$30 \%$ & $23 \%$ & $14 \%$ & $3 \%$ \\
\hline $9 \%$ & $30 \%$ & $21 \%$ & $8 \%$ \\
\hline $57 \%$ & $16 \%$ & $3 \%$ & $12 \%$ \\
\hline $15 \%$ & $30 \%$ & $22 \%$ & $7 \%$ \\
\hline $21 \%$ & $24 \%$ & $10 \%$ & $14 \%$ \\
\hline $27 \%$ & $21 \%$ & $17 \%$ & $6 \%$ \\
\hline $33 \%$ & $26 \%$ & $11 \%$ & $6 \%$ \\
\hline
\end{tabular}

\begin{tabular}{c|c|c}
\multicolumn{3}{c}{ Size } \\
\hline Core & Periph. & X-Periph. \\
\hline $0 \%(41)$ & $25 \%$ & $74 \%$ \\
$0 \%(25)$ & $20 \%$ & $80 \%$ \\
$1 \%(21)$ & $28 \%$ & $72 \%$ \\
$1 \%(17)$ & $26 \%$ & $74 \%$ \\
$0 \%(8)$ & $24 \%$ & $76 \%$ \\
$0 \%(7)$ & $28 \%$ & $72 \%$ \\
$0 \%(3)$ & $32 \%$ & $68 \%$ \\
$0 \%(8)$ & $14 \%$ & $86 \%$ \\
$0 \%(2)$ & $14 \%$ & $86 \%$ \\
\hline $2 \%(13)$ & $30 \%$ & $68 \%$ \\
$0 \%(3)$ & $20 \%$ & $80 \%$ \\
$0 \%(5)$ & $20 \%$ & $80 \%$ \\
$0 \%(2)$ & $21 \%$ & $79 \%$ \\
\hline $0 \%$ & $23 \%$ & $77 \%$
\end{tabular}

Table 2: Posts from the three classes of users; forums are sorted by total number of posts over the life of each forum in descending order. Values are rounded. Highlighting corresponds to magnitude in all cases to improve readability

This resulted in 24 networks. The mean fitness of this set of networks was $\mathrm{R}=.68$. Core sizes were uniformly very small. The Fibromyalgia forum had the largest core of 41 people, which represents roughly $.5 \%$ of the 9361 posters in that forum. In many cases, cores were two or three members and on the order of $.1 \%$ of all posters. Core sizes were correlated with the number of individuals that have dyadic interactions $(\mathrm{R}=.89 ; p<.001)$, indicating that the tendency to engage in dyadic interactions may also reflect a community's chances of forming a core. Somewhat surprisingly, however, overall community size was not with core size $(\mathrm{R}=.34, n . s$. $)$ suggesting that that the development of a core is not inevitable as a community grows. We revisit this observation in the discussion section below.

The preceding analysis segregates the community into three classes of members - the core, operationalized as in [5], the periphery, consisting of those participants that engage in at least one dyadic interaction but do not become part of the core, and the extra-periphery (shortened to x-periphery in the following), consisting of the remaining set of forum participants. We can also segregate paid staff membersincluding both medical experts and staff members-from the other classes. We now examine communication patterns within and among these groups.

In the following analysis, and as discussed above, we further narrowed the set of forums with core-periphery structures to those naming a specific disease or disorder. This yielded the set of thirteen forums shown in Table 2 .

\section{THE RESPONSIBILITY FOR SOCIAL SUPPORT}

Across the identified set of thirteen forums, members of the core have a disproportionately large impact on each forum. Summaries of the proportion of messages between the different classes of users are shown in Table 2 and Figure 1. Top-level posts make up about $22 \%$ of all traffic (note the apparent discrepancy with Table 2 is due to rounding errors). Half of this comes from the x-periphery, which constitutes on average $77 \%$ of the total posting population for each community. Because WebMD is a question and answer style forum, top-level posts are often questions from forum participants. The other roughly $78 \%$ of posting activity takes the form of replies, and the core, which makes up on average $.5 \%$ of each community, is responsible for about a third of these posts. This high proportion of replies by core members is especially pronounced in some communities; for instance, the three community members that make up the core of the HIV/AIDS community generate $34 \%$ of all posts $(11,420$ total over the collection period) in the form of replies.

In summary, the x-periphery, which consists of the largest number of users, also asks the most questions. The core, which is by far the smallest portion of members, is responsible for the vast majority of replies. The periphery tends to lie between these two groups in terms of quantity

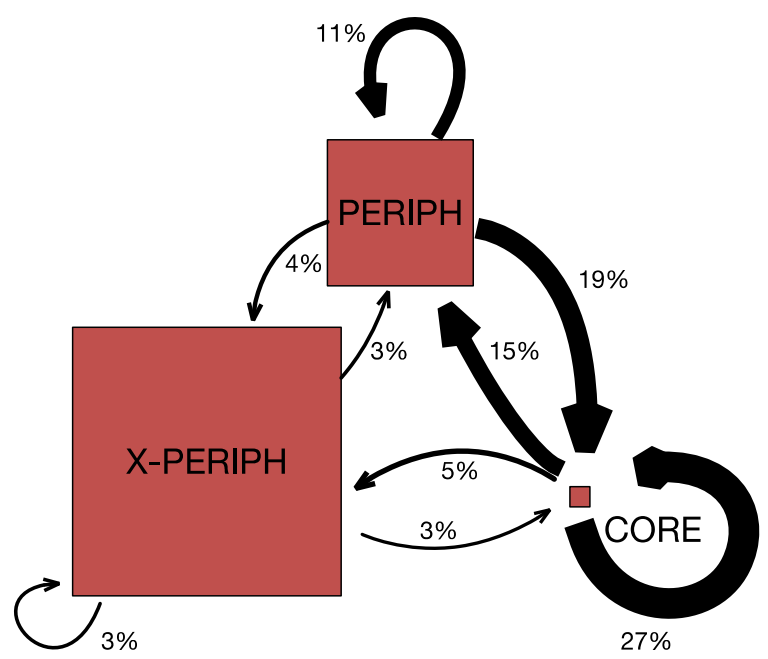

Figure 1: Percentage of replies (top-level posts are excluded) between core, periphery, and extra-periphery. Node area is proportional to number of members in each class. Percentages are of total aggregate reply traffic. Communications to and from staff are omitted, so percentages do not sum to one. 
and type of production.

Examining replies to and from each class as a proportion of the specific source or target's traffic sheds more light on how the different classes allocate effort and who is responsible for meeting their needs (Table 3). More than half the reply-traffic generated by the core is to the core, but it still provides the largest portion of replies to each of the other classes of users. The periphery also plays a significant role for the other classes, but more than half of its replies are directed to the core. The $\mathrm{x}$-periphery replies slightly more to the periphery than any other class, but handles roughly one quarter of its own replies. Hired staff, in general, plays a relatively insignificant role overall.

To appreciate the significance of these patterns, it is useful to consider the average tenure of member-classes. The average length of time between first and last observed posts was 1053 days for core members, 115 days for periphery members, and 13 days for x-periphery. Thus, the core consists primarily of long-term users, who have invested a great deal of time in one another by developing relationships and presumably offering a range of social support over time. The x-periphery (and to a lesser extent, the periphery) consists of a much larger, dynamic population of users who might post once or a handful of times. From the core's perspective, these members are strangers (x-periphery members usually appear once or a handful of times) with needs (they usually ask questions) who probably won't stick around after they find an answer. Yet despite this, and the relatively small size of the cores, they have the capacity to provide $37 \%$ of the replies the $x$ periphery receives from the forum. This is especially notable considering that the paid staff only provides $7 \%$ of the replies to the $\mathrm{x}$-periphery.

The above observations are based solely upon an analysis of the posting rates and message targets among the various classes of users. To gain deeper insight into the kind of support core members provide one another and the other classes of users, we coded a subset of messages for support type, and report the results in the following section.

\section{Kinds of Support}

Wang et al. [54] presented evidence that the degrees of emotional and informational support individuals are exposed to bear a statistical relationship to their tenure in a breast-cancer support community. The authors found that those individuals that are exposed to higher than average levels of emotional support are more likely than average to maintain their membership on the site, and those that are exposed to higher than average levels of informational support are more likely to leave. Additionally, levels of posting activity are also correlated with longer tenures.

As discussed above, member tenure on the site tends to decrease from core to x-periphery. Given that the core is also responsible for a large degree of the replies to others, and in light of the results reported by Wang et al. [54] we hypothesized that the relative amounts of emotional and

\begin{tabular}{r|cccc} 
& \multicolumn{4}{|c}{ Traffic From } \\
\hline Traffic to & Core & Periphery & X-Periphery & Staff \\
\hline Core & $55 \%$ & $50 \%$ & $28 \%$ & $42 \%$ \\
Periphery & $30 \%$ & $31 \%$ & $32 \%$ & $33 \%$ \\
X-Periphery & $10 \%$ & $10 \%$ & $30 \%$ & $21 \%$ \\
Staff & $5 \%$ & $6 \%$ & $9 \%$ & $3 \%$
\end{tabular}

Traffic To

\begin{tabular}{r|cccc}
\hline Traffic from & Core & Periphery & X-Periphery & Staff \\
\hline Core & $54 \%$ & $49 \%$ & $37 \%$ & $46 \%$ \\
Periphery & $37 \%$ & $35 \%$ & $30 \%$ & $36 \%$ \\
X-Periphery & $6 \%$ & $11 \%$ & $24 \%$ & $16 \%$ \\
Staff & $3 \%$ & $4 \%$ & $7 \%$ & $2 \%$
\end{tabular}

Table 3: Proportion of directed traffic by source and target; traffic is proportional on a per column basis. Highlighting corresponds to magnitude to aid readability.

informational support would vary with the target of the reply. Specifically:

H1: The degree of informational support contained in replies from the Core will decrease with the class of the target in order of average tenure on the site; i.e. $X$ Periphery $>$ Periphery $>$ Core

H2: The degree of emotional support contained in replies from the Core will increase with the class of the target in order of average tenure on the site; i.e. $X$ Periphery $<$ Periphery $<$ Core.

Wang et al. [54] did not consider community support in their analysis, although Bambina [2] found that community support tended to be higher among core community members in a breast cancer support forum. From this work, we hypothesized:

H3: The degree of community support contained in replies from the Core will increase with the class of the target in order of more central participation; i.e. XPeriphery $<$ Periphery $<$ Core.

To examine these hypotheses, we partially replicated the coding strategy employed by Wang et al. [54], asking Amazon's Mechanical Turk (MTurk) workers to rate emotional and informational support for individual posts on a seven-point Likert scale. Following Wang et al. [54], we provided workers with the following instructions about emotional and informational support, originally drawn from Bambina [2]:

- Emotional support messages provide understanding, encouragement, affirmation, sympathy, or caring.

- Informational support messages provide advice, referrals or knowledge.

Deviating slightly from the task described by Wang et al. [54], we paid our worker's \$.10 per task (twice that which was paid in [54]) to meet the guidelines for fair wages suggested by [59]. We also used nine MTurk workers per item instead of ten. ${ }^{3}$

${ }^{3}$ As of July 2015, Amazon applies a surcharge to any 


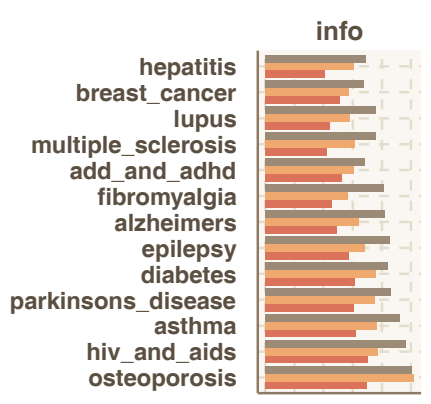

$0 \begin{array}{lllllllll}0 & 2 & 3 & 4 & 5\end{array}$

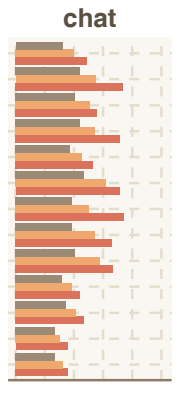

$\begin{array}{llllll}0 & 1 & 2 & 3 & 4 & 5\end{array}$

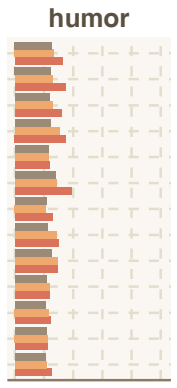

$\begin{array}{llllll}0 & 1 & 2 & 3 & 4 & 5\end{array}$

Support level

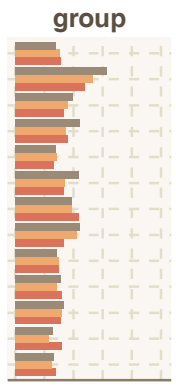

$\begin{array}{llllll}0 & 1 & 2 & 3 & 4 & 5\end{array}$

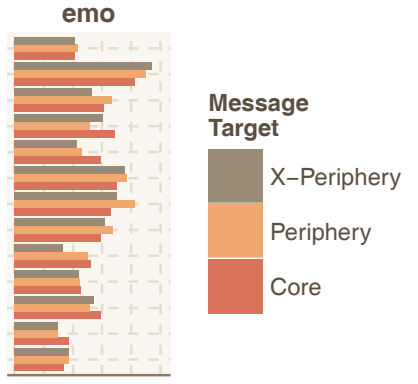

$\begin{array}{llllll}0 & 1 & 2 & 3 & 4 & 5\end{array}$

Figure 2: Different kinds of support delivered by the core to different message targets for each forum; note that $1=$ "none" and $7=$ "a great deal," while 4 is the midpoint. Forums are sorted by mean informational support.

To code for community support, we followed the same procedure as above, adopting the definitions for community support provided by Bambina [2]. Bambina subdivided community support into three subcategories, which include: chatting, humor, and groupness. After a pilot study with graduate research assistants, we found that identifying these kinds of content required distinct analyses, and it was unclear that these categories were well correlated across posts. Therefore, we asked MTurk workers to code for these categories separately, using the following definitions:

- Chatting involves light-hearted conversation that focuses on non-disease related information; e.g. "It's so cold in Buffalo right now!" or "I like to do pilates every morning after I wake up."

- Humor involves jokes or expressions of humor (e.g. LOL) or teasing.

- Groupness involves references to the community itself; e.g. "We are all pulling for you," or "The people here are so supportive," or to a lesser extent, "Let us know how it goes."

As with the prior task, we asked 9 individuals to code each of the posts on a 7-point Likert scale, and paid each worker $\$ .10$ per task.

Posts for coding were drawn from each of the three message targets (from Core to Core, Periphery, or XPeriphery) over each of the 13 forums shown in Table 2, creating a total of 39 categories of message. Only messages responding to a single poster were chosen, but messages were otherwise randomly selected within each category. There were not enough messages in seven of the 39 categories to create an even draw; 56 messages were selected in each of the remaining 32 categories, for a total of 2109 messages coded (652 core, 728 periphery, $728 \mathrm{x}$ periphery).

Results of this analysis by message target are summarized visually in Figure 2, and quantitative analysis is provided Table 3. We used the intraclass correlation coefficient to

MTurk tasks with more than 9 individual Assignments per HIT. evaluate the consistency of the ratings for each type of support [28]. All were high, though the community support ratings were uniformly lower.

To determine how the relative levels of each type of support in a measure varied with message target, we first verified that the level of support was normally distributed for each support type, and then ran separate linear regressions for each type of support. For each regression, support level was modeled as a dependent variable, message target an independent variable, and forum identity as a control variable. Message target was dummy coded with "core" as the reference value. Thus the value for core reported in Table 3 reflects the mean level of support along the Likert scale provided to the coders, and coefficients for the other classes can be interpreted as deviations from that mean. We also ran an analysis of variance for each model to determine the relative impact of forum identify and message target.

There was a clear relationship between the degree of informational support and the class of the message target, with the periphery receiving the largest degree of informational support and the core receiving the least, confirming H1. There is no clear relationship between emotional support and message target, so we did not find support for $\mathrm{H} 2$, but instead observe that the level of emotional support seems to be more a property of each individual forum community than it is of the target of a message.

Our findings regarding community support were mixed. We found support for $\mathrm{H} 3$ in the chatting and humor classes, with the perceived amount of each increasing from periphery to core, although the variance was much larger for chatting than humor. However, the relationship between message target and groupness was much weaker, with messages to the x-periphery containing slightly more than messages to the other classes of users.

In summary, informational support contained in responses from the core increases as the target of the message becomes more distant from the core. This is compatible with Wang et al.'s [54] finding that increasing degrees of 


\begin{tabular}{c|c|cccc|cc} 
Support Class & $\begin{array}{c}\text { ICC2 (random } \\
\text { raters) }\end{array}$ & $\mathbf{R}^{\wedge 2}$ & $\begin{array}{c}\text { Core } \\
\text { (intercept) }\end{array}$ & $\begin{array}{c}\text { Periphery } \\
\text { (coefficient) }\end{array}$ & $\begin{array}{c}\text { X-Periphery } \\
\text { (coefficient) }\end{array}$ & $\begin{array}{c}\mathbf{F} \text { (Message } \\
\text { Target) }\end{array}$ & $\begin{array}{c}\mathbf{F} \text { (Forum } \\
\text { Identity) }\end{array}$ \\
\hline Informational & .89 & $.27^{* * *}$ & $2.24^{* * *}$ & $.71^{* * *}$ & $1.37^{* * *}$ & $209.2^{* * *}$ & $27.9^{* * *}$ \\
Emotional & .87 & $.29 * * *$ & $2.37^{* * *}$ & .03 & -.12 & $3.9 *$ & $71.8^{* * *}$ \\
Chatting & .81 & $.23^{* * *}$ & $2.82^{* * *}$ & $-.53^{* * *}$ & $-1.03^{* * *}$ & $154.67^{* * *}$ & $29.1^{* * *}$ \\
Humor & .80 & $.11^{* * *}$ & $1.37^{* * *}$ & $-.19 * * *$ & -.30 & $55.5^{* * *}$ & $13.0^{* * *}$ \\
Groupness & .79 & $.17^{* * *}$ & $1.34^{* * *}$ & 0.0 & $.14 * *$ & $5.0^{* *}$ & $24.8^{* * *}$
\end{tabular}

Table 1: Columns 1 reports ICC for MTurk workers; columns 2-5 report adjusted $\mathbf{R}^{2}$ and coefficients derived from a linear regression; columns 7-8 report $\mathrm{F}$-measures from analysis of variance

informational support in messages are inversely correlated with tenure on the site. Given this, the lack of support for $\mathrm{H} 2$ is somewhat surprising. However, we note that Wang et al. [54] calculated mean informational and emotional support at the thread level, while we look at only individual, targeted message replies. Therefore, Wang et al.'s analysis [54] contains an implicit assumption that the support received by an individual was an average of the support contained in all posts in a thread preceding that individual's post. This may lead to systematic differences because core members are typically involved in all threads, but peripheral members are primarily involved in short, informationally dense threads. Further investigation will be required to determine if this is the case.

Finally, our findings regarding community support are roughly aligned with Bambina's [2] analysis, although we find an important distinction between "groupness" and the other subtypes of community support we analyzed. Bambina [2] did not make this distinction in her analysis of support exchanged between members. The results reported here help paint of more nuanced picture of the overall sociotechnical mechanism of support provision in WebMD. In the following discussion, we summarize our view of this mechanism, specifically within the context of other online communities.

\section{DISCUSSION}

For a designer of online support platforms, understanding the needs of individuals and the roles they might play is important. However, to design online support platforms that are also sustainable, understanding how the roles of subpopulations intertwine to create stable dynamics is critical. We have sought to articulate such a mechanism.

Almost half of the forums we examined on WebMD exhibited a strong core-periphery structure. Our operationalization of this structure, which sets the bar at a single dyadic interaction, naturally leads us to identify a third class of even more peripheral posters - the $\mathrm{x}$ periphery-who never engage in any dyadic interactions and are by far the largest component of the population visiting the forums.

In our data, cores are small groups with strong relationships. The periphery is harder to characterize. Peripheral members do not typically have strong relationships with each other, and most of their attention is focused on the core. Whether they have a distinct role in the functioning of the support community is a subject for future work. Our best characterization at this point is that the periphery has characteristics of both the core and the $\mathrm{x}$ periphery, and is likely made up of a range of participants who are closer to one side or the other. Yet, despite its relative position in a social network, the periphery does not in any sense "mediate" the interactions of the other two kinds of users.

The x-periphery is distinct and consists of a highly dynamic population that is focused primarily upon asking questions. They occasionally engage with one another, or members of other groups, but never, by definition in our case, engage in relationship creation.

The task of delivering social support is differentially allocated among these three classes of users. The core is responsible for the majority of responses both to itself and the other classes. Furthermore, the degree of informational support contained in messages from the core increases as we move out from the core to the x-periphery. The core can be thought of as an engine that is capable of delivering information to a much larger population.

We did not find significant differences in the levels of emotional support offered by the core to other users. This conflicts with Wang et al.'s [54] findings, and may be because we analyze support delivery at the post level, rather than the thread level. We feel this more realistic in the case of emotional support. In light of their findings, Wang et al [54] speculated that emotional support might be one reason members stay longer in a forum; given our inability to replicate this finding, what keeps the core around?

Our analysis suggests it is the companionship individuals find online. The role of companionship and social relationships have been widely discussed in the context of online health support, especially in light of the potential feelings of isolation that stem from being alone with one's disease [2,3,18]. Prior evidence also suggests that companionship, as distinct from other forms of social support, is better suited for helping individuals cope with the day-to-day stress of managing a chronic disease, which becomes more salient in ongoing relationships [44].

In light of our findings, we propose that, as with other bond-based social media variants, the core stays in a community because that is where their friends are. Moving towards the core, we find more and more familiar interactions among individuals with established histories. Core members put more time and effort into these exchanges than others, and as a result, they are more likely 
to be on the site than other community members.

Thus, while we cannot conclusively say why they are willing to provide informational support to so many others (there are likely a variety of reasons [40]), the amount of time they invest makes them more likely to see and respond to others. Following Butler et al.'s [9] arguments, such responsiveness is likely a signal that encourages new members to ask their questions.

Thus, a small group of people is sustained by and devotes significant energy to maintaining their friendships. In turn, this group uses a fraction of their time to support to a much broader population of itinerant users with support needs, which in turn serves to indicate to an even broader population or lurkers that the forum is worth posting on. Thus, a sociotechnical engine for the delivery of online social support is sustained.

This proposal is surprisingly novel within the specific field of online support. Wang et al [54] speculate about the role of informational and emotional exchange in community sustainability, but omit any discussion of the role strong relationships might play. In cases where community support is explicitly considered, its dual role in binding community and enabling support for many, less involved individuals has not been articulated [2,52]. Finally, as discussed above, Ren at al. [40] offer important and relevant insights about shared identity and social bonds, but do not connect these findings to the delivery of online support.

We cannot say how widespread the patterns we have identified are; not all large forums develop cores, and it will be important to examine these forums in the future. Nonetheless, given prior work (e.g. [2]) we expect this to be a common pattern. If so, several questions for future inquiry are important. First, why some users become part of the core is important for those who would seek to design online support communities with this structure in mind. Are core members born core members, or do they traverse a trajectory from x-periphery inward (e.g. [37] or [39])? The broader context of online support communities adds interesting dimensions to this question. For example, is there a relatively stable proportion of individuals who seek the community support via online relationships? How does this vary with disease characteristics and socioeconomic factors?

Another important set of questions concern a community's capacity for support and the parameters that govern its stability. Is it possible to quantify and identify a fixed point at which a core will dissolve? How large a community can a core of a given size or with certain properties support? Are core-sizes self-limiting due to bandwidth limitations (e.g. [8])?

Finally, it is important to understand how sociotechnical design features interact with the mechanism we have articulated. For instance, how does the involvement of medical experts or moderators affect the stability of the core? Previous work has suggested that this type of intervention can reduce interactivity and promote questionseeking behavior [31,42], and this might impair the activities of the core. Focusing more directly on technical design, what sorts of technical affordances are required for core formation and relationship maintenance, and how should these be balanced against affordances designed for information seeking?

There are likely to be other sociotechnical configurations that enable resilient and effective online support systems. In general we see the identification of such mechanisms as a powerful step in focusing research on questions that are critical for developing principled, deliberate sociotechnical design strategies for sustainable online support communities.

\section{CONCLUSIONS}

Online social support systems can play a critical role for those individuals coping with difficult medical conditions, yet it remains unclear what the right ingredients for creating such systems are.

We have sought to shed light on this problem by elucidating a mechanism that enables large-scale online social support. Our analysis reveals a small, densely connected core of participants who form strong relationships at the center of active support communities. This core is responsible for the largest portion of the reply traffic on the site. Much of this traffic is directed back to the core itself, but a substantial portion is devoted to other, more peripheral members.

We propose that, under the right circumstances, core and periphery can interact to create a self-sustaining community with the capacity to provide social support for thousands of individuals. A critical mechanism for such a community is the exchange of support though a tightly knit web of relationships at the core. This exchange, in turn, creates the capacity for the core to provide informational support to a much larger peripheral group of individuals. When such a core develops, it can provide far more social support than paid staff members. From a resources perspective, the core provides a critical health service that has not traditionally been offered by existing health infrastructures [32].

The identification of mechanisms such as those presented here holds the potential to inform design efforts for building sustainable sociotechnical systems in support of the social good. We hope that our work can serve as a resource for others who find promise in this pursuit.

\section{ACKNOWLEDGEMENTS}

This work was supported by NSF grant VOSS-1422982.

\section{REFERENCES}

1. A. Balali, H. Faili, and M. Asadpour. 2014. A Supervised Approach to Predict the Hierarchical Structure of Conversation Threads for Comments. The Scientific World Journal 2014: e479746. http://doi.org/10.1155/2014/479746 
2. A Bambina. 2007. Online Social Support: The Interplay of Social Networks and Computer-Mediated Communication. Cambria Press.

3. Azy Barak, Meyran Boniel-Nissim, and John Suler. 2008. Fostering empowerment in online support groups. Computers in Human Behavior 24, 5: 18671883. http://doi.org/10.1016/j.chb.2008.02.004

4. SP Borgatti, MG Everett, and LC Freeman. 2002. Ucinet for Windows: Software for Social Network Analysis. Analytic Technologies.

5. Stephen P Borgatti and Martin G Everett. 2000. Models of core/periphery structures. Social Networks 21, 4: 375-395. http://doi.org/10.1016/S03788733(99)00019-2

6. Susan L. Bryant, Andrea Forte, and Amy Bruckman. 2005. Becoming Wikipedian: Transformation of Participation in a Collaborative Online Encyclopedia. Proceedings of the 2005 International ACM SIGGROUP Conference on Supporting Group Work, ACM, 1-10. http://doi.org/10.1145/1099203.1099205

7. R. W. Burgoyne. 2005. Exploring direction of causation between social support and clinical outcome for HIV-positive adults in the context of highly active antiretroviral therapy. AIDS Care 17, 1: 111-124. http://doi.org/10.1080/09540120412331305179

8. Brian S. Butler. 2001. Membership Size, Communication Activity, and Sustainability: A Resource-Based Model of Online Social Structures. Information Systems Research 12, 4: 346-362. http://doi.org/10.1287/isre.12.4.346.9703

9. Brian S. Butler, Patrick J. Bateman, Peter H. Gray, and E. Ilana Diamant. 2014. An attraction-selectionattrition theory of online community size and resilience. Mis Quarterly 38, 3: 699-728.

10. Brian Butler, Lee Sproull, Sara Kiesler, and Robert Kraut. 2002. Community effort in online groups: Who does the work and why. Leadership at a distance: Research in technologically supported work: 171-194.

11. Andrea Civan, David W. McDonald, Kenton T. Unruh, and Wanda Pratt. 2009. Locating patient expertise in everyday life. Proceedings of the ACM 2009 international conference on Supporting group work, ACM, 291-300. Retrieved May 15, 2015 from http://dl.acm.org/citation.cfm?id=1531718

12. Andrea Civan and Wanda Pratt. 2007. Threading Together Patient Expertise. AMIA Annual Symposium Proceedings 2007: 140-144.

13. Kevin Crowston, A. Wiggins, and J. Howison. 2010. Analyzing Leadership Dynamics in Distributed Group Communication. 2010 43rd Hawaii International Conference on System Sciences (HICSS), 1-10. http://doi.org/10.1109/HICSS.2010.62

14. K. Crowston, K. Wei, Q. Li, and J. Howison. 2006.
Core and periphery in Free/Libre and Open Source software team communications. System Sciences, 2006. HICSS'06. Proceedings of the 39th Annual Hawaii International Conference on, 118a-118a. Retrieved December 25, 2012 from http://ieeexplore.ieee.org/xpls/abs_all.jsp?arnumber=1 579526

15. Peter Csermely, András London, Ling-Yun $\mathrm{Wu}$, and Brian Uzzi. 2013. Structure and dynamics of core/periphery networks. Journal of Complex Networks 1, 2: 93-123. http://doi.org/10.1093/comnet/cnt016

16. Mihai Cucuringu, M. Puck Rombach, Sang Hoon Lee, and Mason A. Porter. 2014. Detection of CorePeriphery Structure in Networks Using Spectral Methods and Geodesic Paths. arXiv:1410.6572 [condmat, physics:physics]. Retrieved May 16, 2015 from http://arxiv.org/abs/1410.6572

17. Carolyn E. Cutrona and Daniel W. Russell. 1990. Type of social support and specific stress: Toward a theory of optimal matching. In Social support: An interactional view, B. R. Sarason, I. G. Sarason and G. R. Pierce (eds.). John Wiley \& Sons, Oxford, England, 319-366.

18. K. P. Davison, J. W. Pennebaker, and S. S. Dickerson. 2000. Who talks? The social psychology of illness support groups. The American Psychologist 55, 2: 205-217.

19. M. De Choudhury, W. A Mason, J. M Hofman, and D. J Watts. 2010. Inferring relevant social networks from interpersonal communication. Proceedings of the 19th international conference on World wide web, 301-310.

20. Jerry Finn. 1999. An exploration of helping processes in an online self-help group focusing on issues of disability. Health \& Social Work 24, 3: 220-31.

21. D. Fisher, M. Smith, and H.T. Welser. 2006. You Are Who You Talk To: Detecting Roles in Usenet Newsgroups. Proceedings of the 39th Annual Hawaii International Conference on System Sciences, 2006. HICSS '06, 59b-59b. http://doi.org/10.1109/HICSS.2006.536

22. Mary P. Gallant. 2003. The Influence of Social Support on Chronic Illness Self-Management: A Review and Directions for Research. Health Education \& Behavior 30, 2: 170-195. http://doi.org/10.1177/1090198102251030

23. Preetinder Gill and Whisnant. 2012. A qualitative assessment of an online support community for ovarian cancer patients. Patient Related Outcome Measures: 51. http://doi.org/10.2147/PROM.S36034

24. E. Gleave, H.T. Welser, T.M. Lento, and M.A. Smith. 2009. A Conceptual and Operational Definition of "Social Role" in Online Community. 42nd Hawaii 
International Conference on System Sciences, 2009. HICSS '09, 1-11. http://doi.org/10.1109/HICSS.2009.6

25. Andreea Daniela Gorbatai and M. Piskorski. 2012. Social Structure of Contributions to Wikipedia. Harvard Business School. Retrieved September 23, 2013 from

http://www.wjh.harvard.edu/ hos/papers/AndreeaGorb attai/AndreeaGorbattai.pdf

26. Benjamin H. Gottlieb. 1985. Social Networks and Social Support: An Overview of Research, Practice, and Policy Implications. Health Education \& Behavior 12, 1: 5-22. http://doi.org/10.1177/109019818501200102

27. Mark Granovetter. 1973. The Strength of Weak Ties. Social Science Research Network, Rochester, NY. Retrieved April 28, 2014 from http://papers.ssrn.com/abstract $=1504479$

28. Andrea Hartzler and Wanda Pratt. 2011. Managing the personal side of health: how patient expertise differs from the expertise of clinicians. Journal of medical Internet research 13,3 .

29. Starr Roxanne Hiltz. 1993. The Network Nation: Human Communication Via Computer. MIT Press.

30. James Howison, Andrea Wiggins, and Kevin Crowston. 2011. Validity Issues in the Use of Social Network Analysis with Digital Trace Data. Journal of the Association for Information Systems 12, 12: 767797.

31. Jina Huh. 2015. Clinical Questions in Online Health Communities: The Case of "See your doctor" Threads. Proceeding of the 2015 conference on Computer Supported Cooperative Work, ACM Press.

32. B. H. Kaplan, J. C. Cassel, and S. Gore. 1977. Social support and health. Medical Care 15, 5 SUPPL: 47-58.

33. Chen Lin, Jiang-Ming Yang, Rui Cai, Xin-Jing Wang, and Wei Wang. 2009. Simultaneously Modeling Semantics and Structure of Threaded Discussions: A Sparse Coding Approach and Its Applications. Proceedings of the $32 N d$ International ACM SIGIR Conference on Research and Development in Information Retrieval, ACM, 131-138. http://doi.org/10.1145/1571941.1571966

34. Yunzhong Liu, Feng Chen, and Yi Chen. 2013. Learning Thread Reply Structure on Patient Forums. Proceedings of the 2013 International Workshop on Data Management \&\#38; Analytics for Healthcare, ACM, 1-4. http://doi.org/10.1145/2512410.2512426

35. Diane Maloney-Krichmar and Jenny Preece. 2005. A Multilevel Analysis of Sociability, Usability, and Community Dynamics in an Online Health Community. ACM Trans. Comput.-Hum. Interact. 12, 2: 201-232. http://doi.org/10.1145/1067860.1067864

36. Audris Mockus, Roy T. Fielding, and James D.
Herbsleb. 2002. Two Case Studies of Open Source Software Development: Apache and Mozilla. $A C M$ Trans. Softw. Eng. Methodol. 11, 3: 309-346. http://doi.org/10.1145/567793.567795

37. Katherine Panciera, Aaron Halfaker, and Loren Terveen. 2009. Wikipedians Are Born, Not Made: A Study of Power Editors on Wikipedia. Proceedings of the ACM 2009 International Conference on Supporting Group Work, ACM, 51-60. http://doi.org/10.1145/1531674.1531682

38. David Martin Powers. 2011. Evaluation: from Precision, Recall and F-measure to ROC, Informedness, Markedness and Correlation. Retrieved January 8, 2016 from http://dspace.flinders.edu.au/xmlui/handle/2328/27165

39. J. Preece and B. Shneiderman. 2009. The Reader-toLeader Framework: Motivating technology-mediated social participation. AIS Transactions on HumanComputer Interaction 1, 1: 5.

40. Yuqing Ren, Robert Kraut, and Sara Kiesler. 2007. Applying Common Identity and Bond Theory to Design of Online Communities. Organization Studies 28, 3: 377-408. http://doi.org/10.1177/0170840607076007

41. Yuqing Ren, Robert Kraut, Sara Kiesler, and Paul Resnick. 2012. Encouraging commitment in online communities. Building successful online communities: Evidence-based social design: 77-125.

42. Catherine Ridings and Molly McLure Wasko. 2010. Online discussion group sustainability: Investigating the interplay between structural dynamics and social dynamics over time. Journal of the Association for Information Systems 11, 2. Retrieved from http://aisel.aisnet.org/jais/vol11/iss2/1

43. J. Brian Rollman, Kevin Krug, and Fredrick Parente. 2000. The Chat Room Phenomenon: Reciprocal Communication in Cyberspace. CyberPsychology \& Behavior 3, 2: 161-166. http://doi.org/10.1089/109493100316003

44. Karen S. Rook. 1987. Social support versus companionship: Effects on life stress, loneliness, and evaluations by others. Journal of Personality and Social Psychology 52, 6: 1132-1147. http://doi.org/http://dx.doi.org.proxy2.cl.msu.edu/10.1 037/0022-3514.52.6.1132

45. Jacob Solomon and Rick Wash. 2014. Critical Mass of What? Exploring Community Growth in WikiProjects. Retrieved January 24, 2015 from http://www.aaai.org/ocs/index.php/ICWSM/ICWSM14 /paper/download/8104/8148

46. Margarita Staniute, Julija Brozaitiene, and Robertas Bunevicius. 2013. Effects of Social Support and Stressful Life Events on Health-Related Quality of Life 
in Coronary Artery Disease Patients: The Journal of Cardiovascular Nursing 28, 1: 83-89. http://doi.org/10.1097/JCN.0b013e318233e69d

47. Joni L. Strom and Leonard E. Egede. 2012. The Impact of Social Support on Outcomes in Adult Patients with Type 2 Diabetes: A Systematic Review. Current diabetes reports 12, 6: 769-781. http://doi.org/10.1007/s11892-012-0317-0

48. Bongwon Suh, Gregorio Convertino, Ed H. Chi, and Peter Pirolli. 2009. The Singularity is Not Near: Slowing Growth of Wikipedia. Proceedings of the 5th International Symposium on Wikis and Open Collaboration, ACM, 8:1-8:10. http://doi.org/10.1145/1641309.1641322

49. Shelley E. Taylor. 2011. Social support: A review. In The Oxford Handbook of Health Psychology, Howard S. Friedman (ed.). Oxford University Press, 189-214. Retrieved April 1, 2015 from https://books.google.com/books?hl=en\&lr=\&id=apBo AgAAQBAJ\&oi=fnd\&pg=PA189\&dq=social+support :+a+review+taylor\&ots $=73 \mathrm{zdmsH6vO \& sig=PaBBp6F}$ yaLCBBXf3ZYTtokBcGwQ

50. Van Der Molem. 1999. Relating information needs to the cancer experience: 1 . Information as a key coping strategy. European Journal of Cancer Care 8, 4: 238244. http://doi.org/10.1046/j.1365-2354.1999.00176.x

51. Hongning Wang, Chi Wang, ChengXiang Zhai, and Jiawei Han. 2011. Learning Online Discussion Structures by Conditional Random Fields. Proceedings of the 34th International ACM SIGIR Conference on Research and Development in Information Retrieval, ACM, 435-444. http://doi.org/10.1145/2009916.2009976

52. Xi Wang, Kang Zhao, and Nick Street. 2014. Social Support and User Engagement in Online Health
Communities. In Smart Health, Xiaolong Zheng, Daniel Zeng, Hsinchun Chen, Yong Zhang, Chunxiao Xing and Daniel B. Neill (eds.). Springer International Publishing, 97-110. Retrieved September 5, 2015 from http://link.springer.com/chapter/10.1007/978-3-31908416-9_10

53. Yi-chia Wang, Mahesh Joshi, William Cohen, and Carolyn Rosé. 2008. Recovering Implicit Thread Structure in Newsgroup Style Conversations.

54. Yi-Chia Wang, Robert Kraut, and John M. Levine. 2012. To Stay or Leave?: The Relationship of Emotional and Informational Support to Commitment in Online Health Support Groups. Proceedings of the ACM 2012 Conference on Computer Supported Cooperative Work, ACM, 833-842. http://doi.org/10.1145/2145204.2145329

55. Barry Wellman and Scot Wortley. 1990. Different Strokes from Different Folks: Community Ties and Social Support. American Journal of Sociology 96, 3: 558-588.

56. Howard T. Welser, Eric Gleave, Danyel Fisher, and Marc Smith. 2007. Visualizing the signatures of social roles in online discussion groups, The. Journal of Social Structure: 8.

57. Social Support for Health: An Increased Role in Health Care? | Peers For Progress. Retrieved July 29, 2015 from http://peersforprogress.org/pfp_blog/socialsupport-for-health-an-increased-role-in-health-care/

58. Alexa - Top Sites by Category: Health. Retrieved January 24, 2015 from http:/www.alexa.com/topsites/category/Top/Health

59. Guidelines for Academic Requesters - WeAreDynamo Wiki. Retrieved July 17, 2015 from http://wiki.wearedynamo.org/index.php/Guidelines_for _Academic_Requesters 